\title{
Investigation of the Sound Absorbing Performance of the Hollow Carbon Nanospheres as an Acoustic Absorbent
}

\author{
Mahdi Teymourzadeh ${ }^{1 *}$, Mohammad Seifi ${ }^{2}$ and Ibrahim Hasanzadeh ${ }^{3}$
}

1,2Department of Physics, Faculty of Sciences, University of Guilan, Rasht, Iran.

${ }^{3}$ Reactor and Nuclear Safety Research School, Nuclear Science and Technology Research Institute, Isfahan, Iran.

\author{
*Corresponding Author: \\ $\longrightarrow$ teymourzadehm@gmail.com
}

Received: 10 April, 2021

Accepted: 15 July, 2021

Published: 30 July, 2021

\section{Introduction}

Nowadays, Advances in nanotechnology have provided acoustic researchers with a number of new materials, with features such as nanofibers and nanopores that can potentially be implemented as porous acoustic absorbers. The molecular behavior of these new nanoscopic materials may have a significant influence on their sound absorption properties. In addition, their properties could play an important role in reducing the thickness and mass of absorbers compared with currently available materials. In the current era of nanotechnology, a variety of nanotube constituents are available that can be formed in to nanoscopic fibers, for instance carbon nanotubes (CNTs) [1], boron nitride nanotubes (BNTs) [2] and titania nanotubes (TNAs) [3, 4]. Among the various forms of carbon nanostructures, the hollow carbon nanospheres (HCNSs) have attracted much attention due to their unique structures as well as their inherited properties such as high surface-to-volume ratio, low density, good mechanical strength, good thermal stability, high chemical inactivity, high volume of pores, high surface permeability and reduced transport lengths for both mass and charge transport (short diffusion distance) [5]. In addition, the HCNSs also possess micropores, allowing small molecules to diffuse in and out through the carbon-shell [6]. The HCNSs have been proposed for use in a range of applications including catalyst support, hydrogen storage materials, sensors, nanoelectrodes, semiconductors, supercapacitors, biomaterials, drug delivery, dye encapsulation, contaminated waste removal and so on [7].

However, a fundamental understanding of the mechanisms associated with the use of HCNSs as acoustic absorbers has not been developed, and their potential benefits have not been quantified. Furthermore, measurements of the acoustic absorption properties of HCNSs on their own have not been reported to date. The experimental investigation of the acoustic absorption characteristic of the HCNSs reported in this paper is a first step towards the establishment of a fundamental understanding of acoustic absorption HCNSs materials at the nanoscale. Development of this understanding will advance the knowledge base of the discipline, and will also lay the ground work for other novel arrangements of acoustic absorbers HCNSs to be investigated [4]. 


\section{Acoustic absorption}

The frequency range for human hearing, commonly referred to as audio frequencies, is typically cited as approximately $20 \mathrm{~Hz}-20 \mathrm{KHz}$ [8]. While sensitivity to particular frequencies can be affected by a number of conditions, including age and physical condition, the most important frequencies for understanding speech are typically in the range 500 to $2048 \mathrm{~Hz}$ [9]. Materials and structures that can absorb or attenuate frequencies in this range are important tools in managing the acoustic environment for the health, safety, and comfort of people exposed to noisy environments. Vibration damping and acoustic absorption occur through different mechanisms. Vibration damping occurs when acoustic energy is transmitted directly from contact between two solids. Acoustic absorption occurs when acoustic energy is transmitted through the air and interacts with a solid structure. The morphology of the material is a critical aspect in how it interacts with the air. A material that performs well as a vibration damping material will not necessarily be a good acoustic absorber. This paper will focus on acoustic absorption rather than vibration damping [10]. Sound travels through air in the form of pressure waves. These waves can travel great distances without the individual molecules traveling very far. This occurs through the molecule to molecule transfer of energy
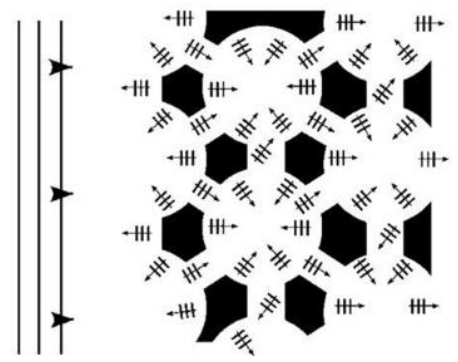

For instance, viscous stresses caused by shearing of the fluid convert fluid kinetic energy into heat. This heat can be transferred from the fluid into a solid structure through heat conduction. Friction on the fluid across the solid also produces heat that can be transferred from the solid to the fluid. Impact of an acoustic pressure wave on a solid structure can dissipate energy through flexing of the solid frame. If the solid surface is nonporous, incident energy reflects back into the environment and is lost. However, if that surface is highly porous, a substantial portion of the pressure wave penetrates the material before encountering a solid surface. The same efficient reflection occurs, but in a structure where the chances are great that the reflected energy will encounter another part of the solid structure before being lost to the environment. A high number of internal reflections can transfer energy to the solid structure through frictional losses and efficiently absorb sound. This scattering is essential to the performance of acoustic absorbers fashioned from materials such as glass fibers, polymers, and metal foams. In order to achieve a large number of interactions, the pressure wave must penetrate deeply enough into the material so as to not immediately be reflected back into the surrounding air. As the pore size decreases, less energy is transferred into the solid structure and more is reflected from the surface, making the material less useful as an acoustic absorber.

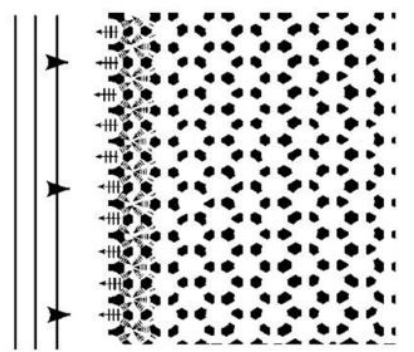

Figure 1. Decrease in energy transfer potential as pore size decreases from large (left) to small (right).

through collisions, allowing the energy to move through the medium. The ability of a material to absorb acoustic energy depends on these pressure waves being able to penetrate a porous solid to a sufficient degree such that energy is dissipated through interaction with the solid portion of the material rather than reflecting back out. Experimental determination of flow resistivity and characteristic impedance allow sound absorbing charts to be constructed that describe the performance of an acoustic absorbing material for various applications. The understanding of the effect on the acoustic properties of a material as the balance between density, speed of sound, and porosity can provide guidance for the development and evaluation of absorber materials. Acoustic energy can be converted into heat through a number of mechanisms.
Porous absorbing materials

Sound-absorbing materials absorb most of the sound energy striking them and reflect very little. Therefore, sound-absorbing materials have been found to be very useful for the control of noise. They are used in a variety of locations: close to sources of noise (close to sources in electric motors, for example), in various paths (above barriers), and sometimes close to a receiver (inside earmuffs).A wide range of soundabsorbing materials exist; they provide absorption properties dependent upon frequency, composition, thickness, surface finish, and method of mounting. 
However, materials that have a high value of sound absorption coefficient are usually porous [11]. A porous absorbing material is a solid that contains cavities, channels or interstices so that sound waves are able to enter through them. It is possible to classify porous materials according to their availability to an external fluid such as air. Figure 2 shows a schematic cross-section of a porous solid material. Those pores that are totally isolated from their neighbors are called closed pores. They have an effect on some macroscopic properties of the material such as its bulk density, mechanical strength and thermal conductivity. However, closed pores are substantially less efficient than open pores in absorbing sound energy. On the other hand, open pores have a continuous channel of communication with the external surface of the body, and they have great influence on the absorption of sound. Open pores can also be blind (open only at one end) or through (open at two ends). A practical convention is used to make a distinction between porosity and roughness, which assumes that a rough surface is not porous unless it has irregularities that are deeper than they are wide [12]. Porous absorbing materials can be classified as cellular, fibrous, or granular; this is based on their microscopic configurations. Porous materials are characterized by the fact that their surfaces allow sound waves to enter the materials through a multitude of small holes or openings [13].
The TEM image of the HCNSs is shown in Figure 3a. From the SEM image, it can be seen that these capsules have a spherical morphology with an excellent interconnected nanostructure (see Figure 3b). Fig. 3(c, d) shows topographic AFM images and AFM image profile that give an idea about the carbon shell sizes. The average carbon shell sizes is less than $90 \mathrm{~nm}$.

\section{Constructed sample of sound absorber from HCNSs}

In the preparation of the absorber sample, the HCNSs powder was hot-pressed in a mould to obtain a disk shape. The pressure given are just enough for the sample to take shape. This is to retain the porosity of the sample. The constructed absorber sample has diameter of $22 \mathrm{~mm}$ with thickness of $2 \mathrm{~mm}$ (as seen in Figure 4) to fit in the impedance tube diameter in the sound absorption test.

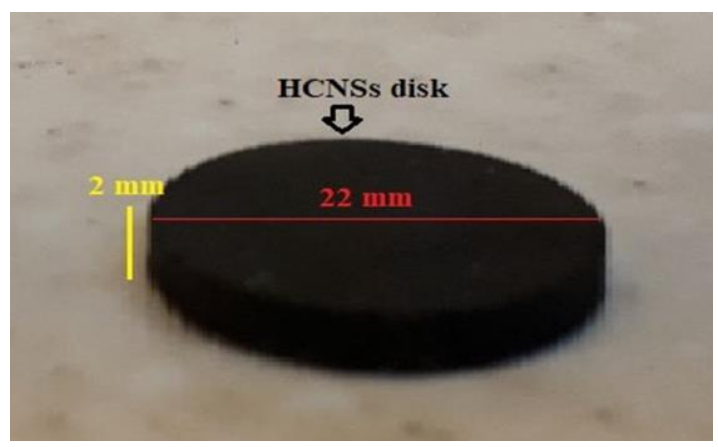

Figure 4. Fabricated sample of the HCNSs

Table 1

Information about the steps of the synthesized HCNSs by CVD method (for detailed information see our previous publication [5]).

\begin{tabular}{llcccc}
\hline SI.No & Materials & $\mathbf{t}(\mathbf{m i n})$ & $\mathbf{T}\left(\mathbf{c}^{\mathbf{0}}\right)$ & Result & Average nanoparticles sizes (nm) \\
\hline 1 & $\mathrm{HgO}+\mathrm{H} 2 \mathrm{O} 2$ & 30 & 400 & $\mathrm{HgO} / \mathrm{C}$ & $\sim 250$ \\
2 & $\mathrm{HgO} / \mathrm{C}$ & 90 & 700 & $\mathrm{HCNSs}$ & 250 \\
\hline
\end{tabular}

\section{Materials and Methods}

\section{HCNSs}

The HCNSs samples were manufactured by a research team in the Nano Laboratory at the University of Guilan, Iran, and also reported in our research team previous publication [5]. The characteristics of these materials are tabulated in table 1. The HCNSs were produced by a CVD method and with a cost-effective method by straight carbonization without any of the additional steps and chemicals [5]

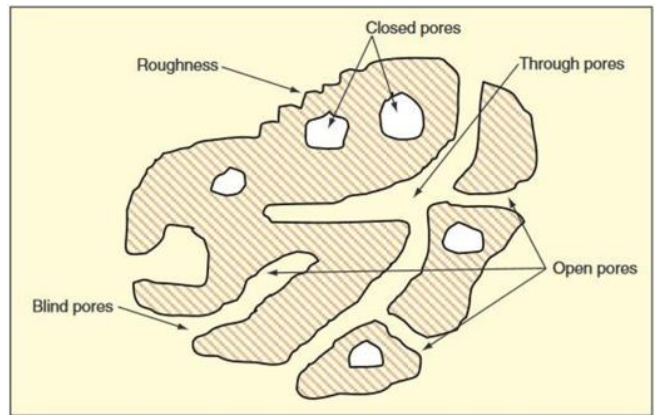

Figure 2. Schematic cross-section of a porous solid material 

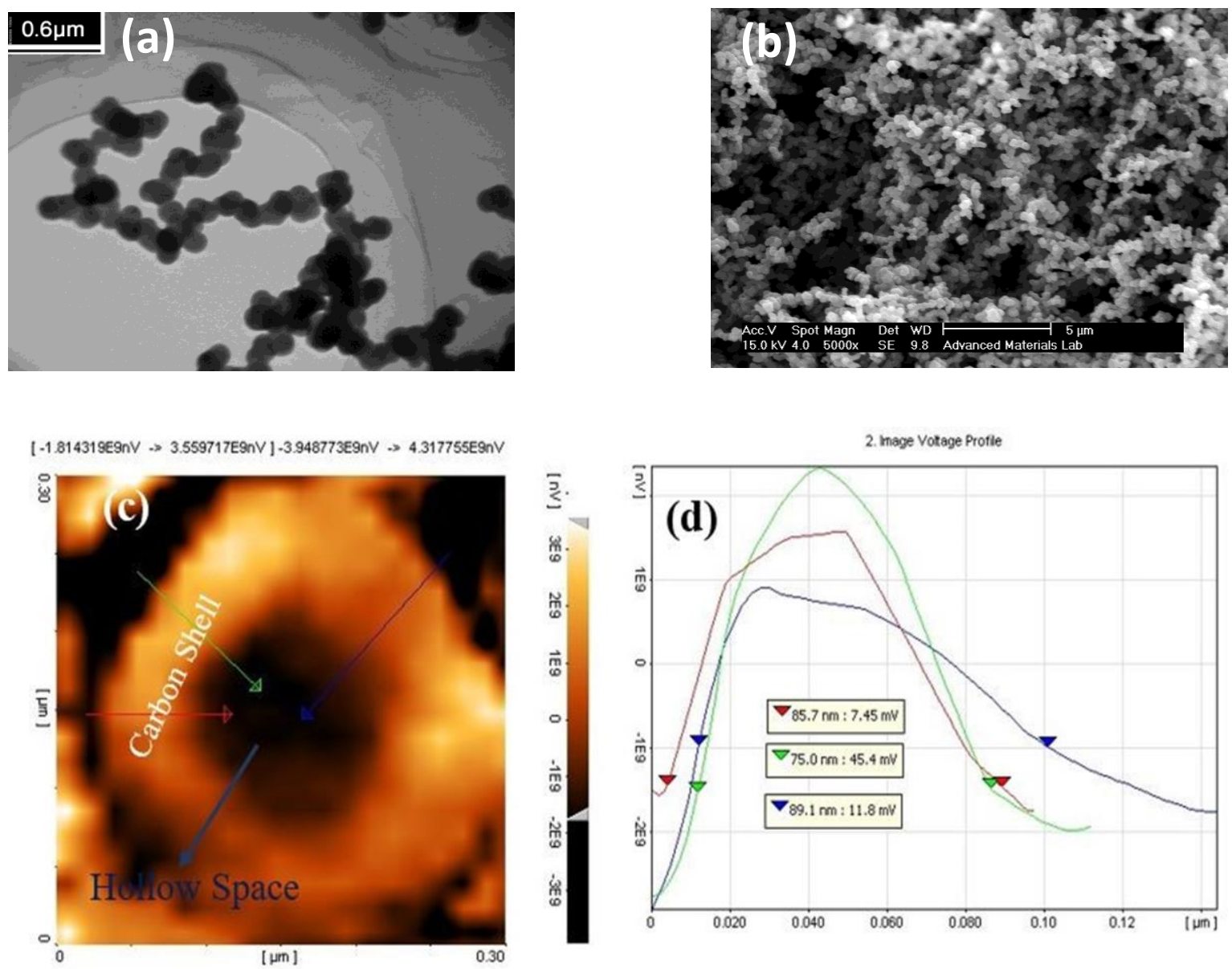

Figure 3. (a) TEM micrographs of HCNSs, (b) SEM images of hollow carbon capsules, (c) AFM image for an individual broken spherical hollow carbon, (d) AFM image profile, the size distribution of the carbon shell (see Figure 3c) is in the range of $75 \mathrm{~nm}$ to $90 \mathrm{~nm}$.

\section{Impedance Tube Apparatus}

The acoustic absorption coefficient of the HCNSs was measured in an impedance tube using two microphones transfer function method in accordance with the ISO 10534-2:2001 [14]. RT Pro Photon v6.34 analyzer with Dactron software was used as the data acquisition system. The signal processing of the measured data was done using Matlab. With diameter of the tube i.e. 22 $\mathrm{mm}$, the reliable frequency range for this experiment is between $500 \mathrm{~Hz}$ to $4.5 \mathrm{kHz}$.

A schematic of the experimental apparatus is shown in Figure 5. The sample was placed at the end of the impedance tube and backed by a rigid surface. The loudspeaker feeds the white noise signal in the tube where the incident and reflected sound pressure recorded by the microphones are then processed to have the absorption coefficient of the sample.

\section{Results and Discussion}

According to Figure 5, the prepared sample was placed at the end of the impedance tube and backed by a rigid surface. The loudspeaker feeds the white noise signal in the tube where the incident and reflected sound pressure recorded by the microphones are then processed to have the absorption coefficient of the sample. Figure 6 plot the acoustic absorption of the 1 gram HCNSs. According to, the absorption coefficient is maximum $(\sim 52 \%)$ at frequency $1500-1700 \mathrm{~Hz}$. Good and stable performance of sound absorption coefficient more than $27 \%$ is shown for frequency 4500-6500 Hz.

According to a report by M. Ayub, A. C.Zander, on the carbon nanotubes (CNTs) acoustic absorption, the CNT acoustic absorption coefficient is observed between $23 \%$ - 28\% at frequency $3000 \mathrm{~Hz}-4000 \mathrm{~Hz}$ (see Figure 7) [15]. These results demonstrate the significance of the sound absorption capability of the HCNSs adsorbents in comparison to CNTs' sound absorption performance. 


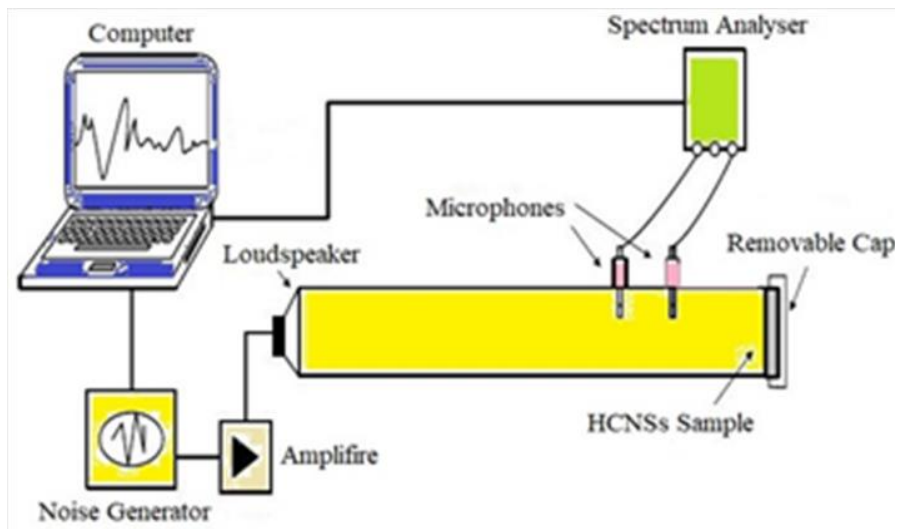

Figure 5. Measurement setup for the sound absorption test

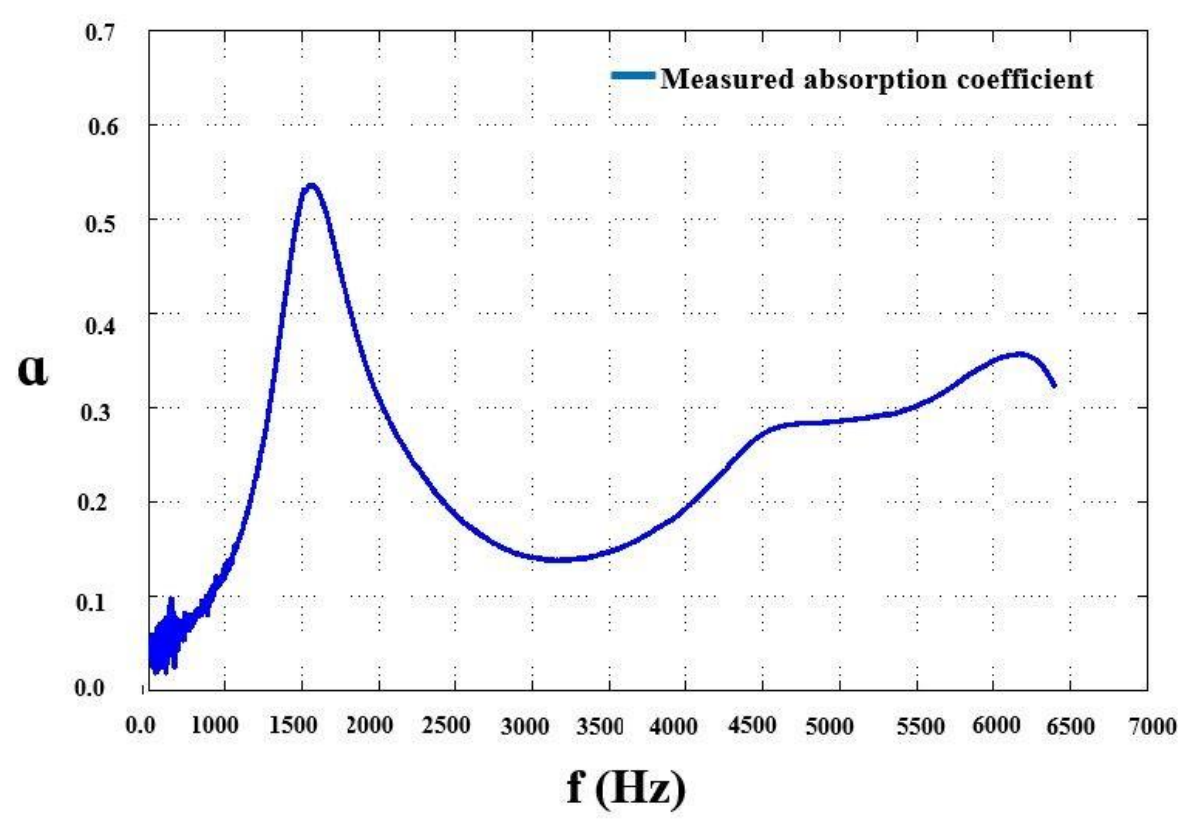

Figure 6. Measured absorption coefficient of the HCNSs

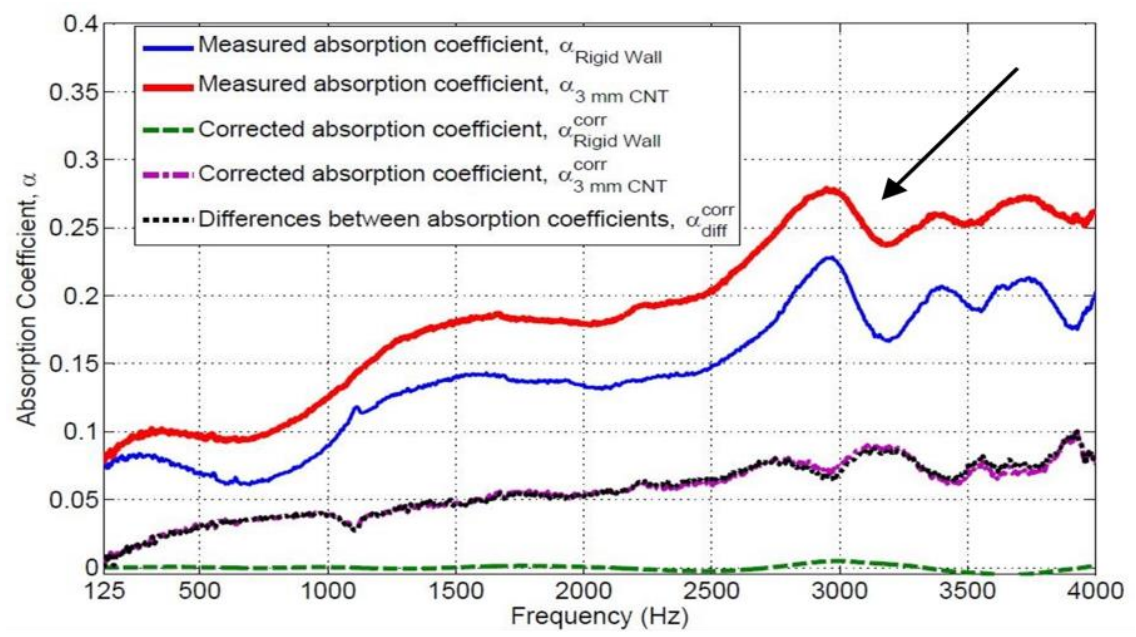

Figure 7. Measured sound absorption coefficient (without correction) of CNT sample (red curve) (adapted from [15]) 


\section{Conclusion}

This article presents the results from an experimental investigation of the acoustic absorption coefficient of the HCNSs using an impedance tube. Results showed the prepared HCNSs sample can provide as much as $50 \%-53 \%$ acoustic absorption at frequency $1500-$ $1700 \mathrm{~Hz}$, and $27 \%-35 \%$ at frequency $4500-6250$ Hz. it is worth noting that the typical carbon available in the nanomaterial lab was used for the investigation without specific optimization for the acoustic absorption behavior. Hence, further investigations would be required to choose the types and arrangement of carbon as a new sound absorber. The acoustic absorption mechanisms and the acoustic behavior of the HCNSs are expected to differ with those of conventional porous materials based on considerations of the physical structure and size of the HCNSs. Therefore, it is of interest to analyses the acoustic behavior using other types of the HCNSs, and HCNSsbased composites.

\section{References}

1. Iijima S. Helical microtubules of graphitic carbon. Nat. 1991; 354(6348): 56-58. Boguslawski L. Influence of pressure fluctuations distribution on local heat transfer on flat surface impinged by turbulent free jet. Proceedings of International Thermal Science Seminar II, Bled, June 2004; 13-16.

2. Koziol K, Vilatela J, Moisala A, Motta M, Cunniff P, Sennett M, Windle A. High performance carbon nanotube fiber. Sci. 2007; 318(5858): 1892-1895.

3. Cohen ML, Zettl A. The physics of boron nitride nanotubes. Phys Today. 2010; 63(11): 34-38.

4. Ayub M, Zander AC, Howard CQ, Cazzolato BS, Huang DM, Shanov VN, Alvarez NT. Normal incidence acoustic absorption characteristics of a carbon nanotube forest. Appl Acoust. 2017; 127: 223-
239.

5. Teymourzadeh M, Seifi M, Hasanzadeh I. Synthesis and characterization of core-shell $\mathrm{HgO} / \mathrm{C}$ colloids and hollow carbon nanospheres by chemical vapor deposition and investigation of its conductivity properties. Solid State Sci. 2018; 84: 95-103.

6. Deshmukh AA, Mhlanga SD, Coville NJ. Carbon spheres. Mater Sci Eng Rep. 2010; 70(1-2): 1-28.

7. Atchudan R, Perumal S, Edison TNJI, Lee YR. Facile synthesis of monodisperse hollow carbon nanospheres using sucrose by carbonization route. Mater Lett. 2016; 166: 145-149.

8. Smith SW. The scientist and engineer's guide to digital signal processing. 1997.

9. Strobel J, Wigley E, Evans N. BUZZ. Acoustical engineering methodologies to measure student engagement in research in engineering education symposium. Palm Cove, Queensland, Australia. 2009.

10. Kuczmarski MA, Johnston JC. Acoustic absorption in porous materials. 2011.

11. Crocker MJ (Ed.). Handbook of noise and vibration control. John Wiley \& Sons. 2007.

12. Rouquerol J, Avnir D, Fairbridge CW, Everett DH, Haynes JM, Pernicone N, Unger KK. Recommendations for the characterization of porous solids (Technical Report). Pure Appl Chem. 1994; 66(8): 1739-1758.

13. Arenas JP, Crocker MJ. Recent trends in porous sound-absorbing materials. Sound Vibrat. 2010; 44(7): 12-18.

14. ISO 10534-2. Acoustic-determination of sound absorption coefficient and impedance tubes-part 2: transfer function method. 2001.

15. Ayub M, Zander AC, Howard CQ, Cazzolato BS, Shanov VN, Alvarez NT, Huang DM. Acoustic absorption behaviour of carbon nanotube arrays. In Inter-noise and Noise-Con congress and conference proceedings. Instit Noise Contr Eng. 2014; 249(7): 929938.

\section{SJFST}

Copyright: (C) 2021 The Author(s); This is an open-access article distributed under the terms of the Creative Commons Attribution License (http://creativecommons.org/licenses/by/4.0), which permits unrestricted use, distribution, and reproduction in any medium, provided the original work is properly cited.

Citation: Teymourzadeh M, Seifi M, Hasanzadeh I. Investigation of the Sound Absorbing Performance of the Hollow Carbon Nanospheres as an Acoustic Absorbent. SJFST, 2021; 3(3):10-15.

https://doi.org/10.47176/sjfst.3.3.10 